\title{
Peripheral Endocannabinoid System Dysregulation in First-Episode Psychosis
}

\author{
Miquel Bioque*,', Borja García-Bueno ${ }^{2,3}$, Karina S MacDowell ${ }^{2,3}$, Ana Meseguer', Pilar A Saiz ${ }^{4}$, \\ Mara Parellada ${ }^{5}$, Ana Gonzalez-Pinto ${ }^{6}$, Roberto Rodriguez-Jimenez ${ }^{3}$, Antonio Lobo ${ }^{7}$, Juan C Leza ${ }^{2,3}$, \\ Miguel Bernardo*,1,8 and From the FLAMM-PEPs study-Centro de Investigación Biomédica en Red de Salud \\ Mental (CIBERSAM) ${ }^{9}$
}

'Schizophrenia Clinic Unit, Neuroscience Institute, Hospital Clinic de Barcelona, Barcelona, Spain; ${ }^{2}$ Department of Pharmacology, Faculty of Medicine, Universidad Complutense de Madrid, Madrid, Spain; ${ }^{3}$ Instituto de Investigación, Hospital 12 de Octubre (i I I), Madrid, Spain;

${ }^{4}$ Department of Psychiatry, Faculty of Medicine, University of Oviedo, Oviedo, Spain; ${ }^{5}$ Child and Adolescent Psychiatry Department, IIS Gregorio Marañón, Hospital General Universitario Gregorio Marañón, Madrid, Spain; ${ }^{6}$ Hospital Universitario de Álava (sede Santiago), Universidad Nacional de Educación a Distancia, Vitoria, Spain; ${ }^{7}$ Department of Psychiatry. Hospital Clínico Universitario and University of Zaragoza, Zaragoza, Spain; ${ }^{8}$ Department of Psychiatry and Clinical Psycobiology, University of Barcelona, Institut d'Investigacions Biomèdiques August Pi i Sunyer (IDIBAPS), Barcelona, Spain

Several hypotheses involving alterations of the immune system have been proposed among etiological explanations for psychotic disorders. The endocannabinoid system (ECS) has a homeostatic role as an endogenous neuroprotective and anti-inflammatory system. Alterations of this system have been associated with psychosis. Cannabis use is a robust risk factor for these disorders that could alter the ECS signalling. In this study, 95 patients with a first episode of psychosis (FEP) and 90 healthy controls were recruited. Protein expression of cannabinoid receptor 2 (CB2), the protein levels of the main endocannabinoid synthesizing enzymes $\mathrm{N}$-acyl phosphatidylethanolamine phospholipase (NAPE) and diacylglycerol lipase (DAGL), and of degradation enzymes fatty acid amide hydrolase (FAAH) and monoacylglycerol lipase (MAGL) were determined by western blot analysis in peripheral blood mononuclear cells (PBMCs). Patients with a FEP showed a decreased expression of CB2 and of both endocannabinoids synthesizing enzymes (NAPE and DAGL) in comparison to healthy controls. After controlling for age, gender, body mass index, and cannabis use, NAPE and DAGL expression remained significantly decreased, whereas FAAH and MAGL expression were increased. On the other hand, FEP subjects with history of severe cannabis use showed a larger ECS dysregulation compared with healthy controls. These results indicate an ECS dysregulation in PBMC of FEP patients. The alteration of the ECS presented at the initial phases of psychosis could be contributing to the pathophysiology of the disease and constitutes a possible biomarker of psychotic disorders and an interesting pharmacological target to take into account for therapeutic purposes.

Neuropsychopharmacology (2013) 38, 2568-2577; doi:I0.1038/npp.20।3.165; published online 31 July 2013

Keywords: biomarker; cannabis; endocannabinoid system; first episode of psychosis; schizophrenia

\section{INTRODUCTION}

Nowadays, we are living a reformulation of the classical concept of the psychotic illness (Insel, 2010), being seen as

*Correspondence: Professor Miguel Bernardo or Miquel Bioque, Unitat d'Esquizofrènia Clínic, Neuroscience Institute, Hospital Clínic de Barcelona, CIBERSAM (G04), C. Villarroel 170. Esc 12. Pta 0, 08036 Barcelona, Spain, Tel: +34 932275400 Ext. 3142, Fax: +34 932275548, E-mail: bernardo@clinic.ub.es or mbioque@clinic.ub.es

${ }^{9}$ FLAMM-PEPs is a multicentric, collaborative and translational group inside CIBERSAM aimed to study inflammatory pathways in psychosis both as possible biomarkers and as possible new therapeutic targets, incorporated in the PEPs study, a Spanish research project in first episodes of psychosis.

Received 28 March 2013; revised 27 May 2013; accepted 25 June 2013; accepted article preview online 4 July 2013 an heterogeneous disorder with a multisystemic impact from the beginning, in addition to its psychiatric expression (Kirkpatrick, 2009). Despite the growing number of published research studies in recent years, the etiology of psychotic disorders is far from being clarified (Bernardo and Bioque, 2010). The exposure to certain environmental factors interacting with genetic factors can alter dopaminergic transmission, neuroendocrine, and cognitive functioning, patterns of interpersonal interaction and affective processing and may lead to a psychopathology worsening (Caspi et al, 2005).

Among other pathophysiological alterations, several hypotheses involving the immune system-at both peripheral and central nervous system (CNS) - have been proposed as etiological explanations for psychosis (GarciaBueno et al, 2013; Garcia-Rizo et al, 2012; Meyer et al, 2011). 
The endocannabinoid system (ECS) has been proposed as a main homeostatic system implicated in the regulation of the complex neuroimmune interactions in diverse neuropathological scenarios (Wolf et al, 2008). In particular, the ECS is present in stress-responsive neural and peripheral circuits, reducing both neurodegenerative and inflammatory damage (Centonze et al, 2008; Wolf et al, 2008).

In short, the ECS refers to the arachidonate-based lipids anandamide (AEA) and 2-arachidonoylglycerol (2AG); their cannabinoid G protein-coupled receptors, namely CB1 and CB2, the two main synthesis enzymes $N$-acyl phosphatidylethanolamine phospholipase (NAPE) and diacylglycerol lipase (DAGL), and the enzymes fatty acid amide hydrolase (FAAH) and monoacylglycerol lipase (MAGL) are responsible for their degradation or reuptake.

Several studies have related the ECS with psychotic disorders (particularly in schizophrenia), focusing on CB1/ CB2 receptors. Reduced $\mathrm{CB} 1$ expression and activity have been found in different brain areas of patients with schizophrenia (Eggan et al, 2008). A close relationship has also been reported between a diminished CB2 function (polymorphism Q63R) and an increased susceptibility to schizophrenia, together with other risk factors (Ishiguro et al, 2010). Schizophrenia symptom remission has been linked to significant changes in CB2 mRNA transcripts in peripheral blood mononuclear cells (PBMC; De Marchi et al, 2003). Moreover, deletion of CB2 has been related to schizophrenia-like behaviors in animal models (OrtegaAlvaro et al, 2011). Therefore, it has been reported that both receptors have a homeostatic role in certain situations and their altered expression has been described in patients with schizophrenia: CB1 mainly in the CNS and CB2 at the peripheral level (Hillard et al, 2012). Regarding other components of the ECS, cerebrospinal fluid (CSF) AEA levels have been found elevated in subjects with schizophrenia (Giuffrida et al, 2004; Leweke et al, 1999).

On the other hand, cannabis is one of the most important and studied enviromental risk factors related to psychosis (Torrey et al, 2012). Around 25-50\% of subjects who suffer a first episode of psychosis (FEP) present with cannabis use (Koskinen et al, 2009; Volkow, 2009). Its use in youth increases the risk of developing psychosis, with an estimated odds ratio of 2.10-2.93 (Henquet et al, 2005; Moore et al, 2007), decreasing the age of schizophrenia onset (Sugranyes et al, 2009). The neurobiological mechanisms underlying this increased psychosis susceptibility are poorly understood (D'Souza et al, 2009; Gage et al, 2013). However, some studies have found that frequent cannabis exposure may downregulate AEA signalling in patients with schizophrenia, but not in healthy individuals (Leweke et al, 2007). It has also been described that FEP patients who use cannabis present cognitive impairment associated to altered brain structure in particular areas rich in CB1 (Bangalore et al, 2008; Ho et al, 2011).

Based on these data, we hypothesized that the ECS may be disrupted in the FEP. We aimed to study the expression of the main ECS components in PBMC samples from healthy controls and FEP patients, taking advantage of a Spanish multicenter, longitudinal, naturalistic, follow-up study (PEPs study, from the Spanish abbreviation of Primeros Episodios Psicóticos; Bernardo et al, 2013).
Multiple logistic regression analyses were conducted to identify potential risk/protective factors for suffering a FEP among the ECS components studied. Finally, further statistical analyses were conducted to find possible differences in the ECS status according to prolonged, heavy cannabis use.

\section{SUBJECTS AND METHODS}

\section{Subjects}

Six Spanish university hospitals recruited 95 FEP patients and 90 matched controls from September 2010 to April 2011 (see Supplementary Data 1 for details). The inclusion criteria were described and discussed in previous articles (Bernardo et al, 2013; Garcia-Bueno et al, 2013). Inclusion criteria for patients were: (1) age between 9 and 35 years; (2) duration of the psychotic symptoms of less than a year; (3) speak Spanish correctly. The exclusion criteria were: (1) mental retardation, including not only an IQ below 70 but also impaired functioning; (2) history of traumatic head injury with loss of consciousness; (3) history of organic disease with mental repercussions. Healthy controls were selected from the same geographic areas following a pairwise matching. Their inclusion criteria were: (1) same gender as patients; (2) similar age $( \pm 10 \%)$; (3) similar parental socioeconomic status $( \pm 1$ level in the Hollingshead-Redlich scale; Hollingshead and Redlich, 1958); (4) no past or present psychiatric disorder per DSM-IV criteria (American Psychiatric Association, 1994); (5) speak Spanish correctly; (6) no history of psychotic disorder among first-degree relatives. The exclusion criteria for controls were the same than for patients.

The study was approved by the ethics committees of all the participating hospitals. The subjects participated after providing a written, informed consent. In underage subjects, informed consent was signed by legal guardians.

\section{Clinical Assessment}

The clinical assessment in the PEPs study was detailed described in a previous article (Bernardo et al, 2013). Briefly, the diagnosis was established by the semi-structured diagnostic interviews according to DSM-IV criteria (SCID; First et al, 1999). The psychopathological assessment was performed using validated Spanish versions of the Positive and Negative Syndrome Scale (PANSS; Kay et al, 1987), the Young Mania Rating Scale (Young et al, 1978), and the Montgomery-Asberg Depression Rating Scale (MADRS; Montgomery and Asberg, 1979). The Global Assessment of Functioning Scale (GAF) and the Children's Global Assessment Scale were used to measure the global severity of symptoms and the level of functioning (Endicott et al, 1976; Shaffer et al, 1983). We calculated the potency equivalents to Chlorpromazine of every antipsychotic dosage, following the international consensus (Gardner et al, 2010). Apart from the interviews with the patient, multiple sources of information (including medical records and interviews with relatives) were used to establish the onset of positive psychotic symptoms (defined as the first week with the PANSS items P1, P3, P5, P6, or G9 scoring four or more). The duration of untreated psychosis was defined as the 
number of days elapsed between this onset and the beginning of the first adequate treatment for psychosis.

Clinical assessment included a complete medical history and physical examination, laboratory tests, and body mass index $\left(\mathrm{BMI}=\right.$ weight in $\mathrm{kg} /$ height in $\left.\mathrm{m}^{2}\right)$. Cannabis use was evaluated by a portion of the European Adaptation of a Multidimensional Assessment Instrument for Drug and Alcohol Dependence (EuropAsi; Kokkevi and Hartgers, 1995). A systematic recording of drug misuse habits was performed.

\section{Biochemical Determinations in PBMC}

Blood sample collection, preparation, and cytosolic extraction conditions were described in a previous article (GarciaBueno et al, 2013).

Preparation of cytosolic extracts. PBMCs were homogenized in $150 \mu \mathrm{l}$ buffer $(10 \mathrm{mmol} / \mathrm{l} \mathrm{N}-2$-hydroxyethylpiperazine- $N^{\prime}$-2-ethanesulfonic acid ( $\left.\mathrm{pH} 7.9\right) ; 1 \mathrm{mmol} / \mathrm{l}$ EDTA, 1 phenylmethylsulfonyl fluoride, $0.1 \mathrm{mg} / \mathrm{ml}$ aprotinin, $1 \mathrm{mg} / \mathrm{ml}$ leupeptin, $1 \mathrm{mg} / \mathrm{ml}$ Na-p-tosyl-L-lysine-chloromethyl ketone, $5 \mathrm{mmol} / \mathrm{l} \mathrm{NaF}, 1 \mathrm{mmol} / \mathrm{l} \mathrm{NaVO}_{4}, 0.5 \mathrm{~mol} / \mathrm{l}$ sucrose, and $\left.10 \mathrm{mmol} / 1 \mathrm{Na}_{2} \mathrm{MoO}_{4}\right)$. After $15 \mathrm{~min}$, Nonidet P-40 (Roche, Mannheim, Germany) was added to reach a concentration level of $1 \%$. The tubes were vortexed for $30 \mathrm{~s}$, and nuclei were collected by centrifugation at $8000 \mathrm{~g}$ for $5 \mathrm{~min}$. The supernatants were considered to be the cytosolic fraction, being stored at $-80^{\circ} \mathrm{C}$. All steps of the fractionation were carried out at $4{ }^{\circ} \mathrm{C}$. As an analysis of their purity, cytosolic extracts were assayed by western blot (WB) analysis against GAPDH, SP-1, or $\beta$-actin (in cytosol:
$99 \pm 1 ; 19 \pm 5 ; 98 \pm 1 \%$ of total optical density signal, respectively).

WB analysis. After determining and adjusting protein levels using the Bradford method, extracts were mixed with Laemmli sample buffer (Bio-Rad, USA; SDS 10\%, distilled $\mathrm{H}_{2} \mathrm{O}, 50 \%$ glycerol, $1 \mathrm{M}$ Tris $\mathrm{HCl}, \mathrm{pH}$ 6.8, dithiothreitol and bromophenol blue) with $\beta$-mercaptoethanol $(50 \mu \mathrm{l} / \mathrm{ml}$ Laemmli) and $12.5 \mu \mathrm{g}$ were loaded into an electrophoresis gel. Once separated on the basis of molecular weight, proteins from the gels were blotted onto a nitrocellulose membrane (Amersham Ibérica, Spain) with a semi-dry transfer system (Bio-Rad) and were incubated with specific antibodies: (1) rabbit polyclonal CB2 in a dilution of $1: 1000$ in TBS-Tween (101550; Cayman Chemical); (2) rabbit polyclonal CB1 in a dilution of $1: 750$ in TBS-Tween (ab23703; Abcam); (3) rabbit polyclonal NAPE-PLD in a dilution of $1: 1000$ in TBS-Tween (10306; Cayman Chemical); (4) rabbit polyclonal DAGL $\alpha$ in a dilution of $1: 1000$ in TBS-Tween (sc-133307; Santa Cruz Biotechnology, USA); (5) rabbit polyclonal FAAH in a dilution of $1: 750$ in TBSTween (101600; Cayman Chemical); (6) rabbit polyclonal MAGL in a dilution of $1: 1000$ in 5\% skimmed milk in TBS-Tween (100035; Cayman Chemical); (7) $\beta$-actin mouse monoclonal in a dilution of $1: 15000$ (Clone AC-15; Sigma, Spain); (8) SP1 rabbit polyclonal antibody in a dilution of 1:2000 (sc-59; Santa Cruz Biotechnology); (9) GAPDH monoclonal antibody at 1:5000 (ab9484; Abcam, UK). Membranes were incubated with the respective HRP-linked secondary antibodies $(1: 2000$ in TBS-Tween). Blots were imaged using an Odyssey Fc System (Li-COR Biosciences) and were quantified by densitometry (NIH ImageJ software).
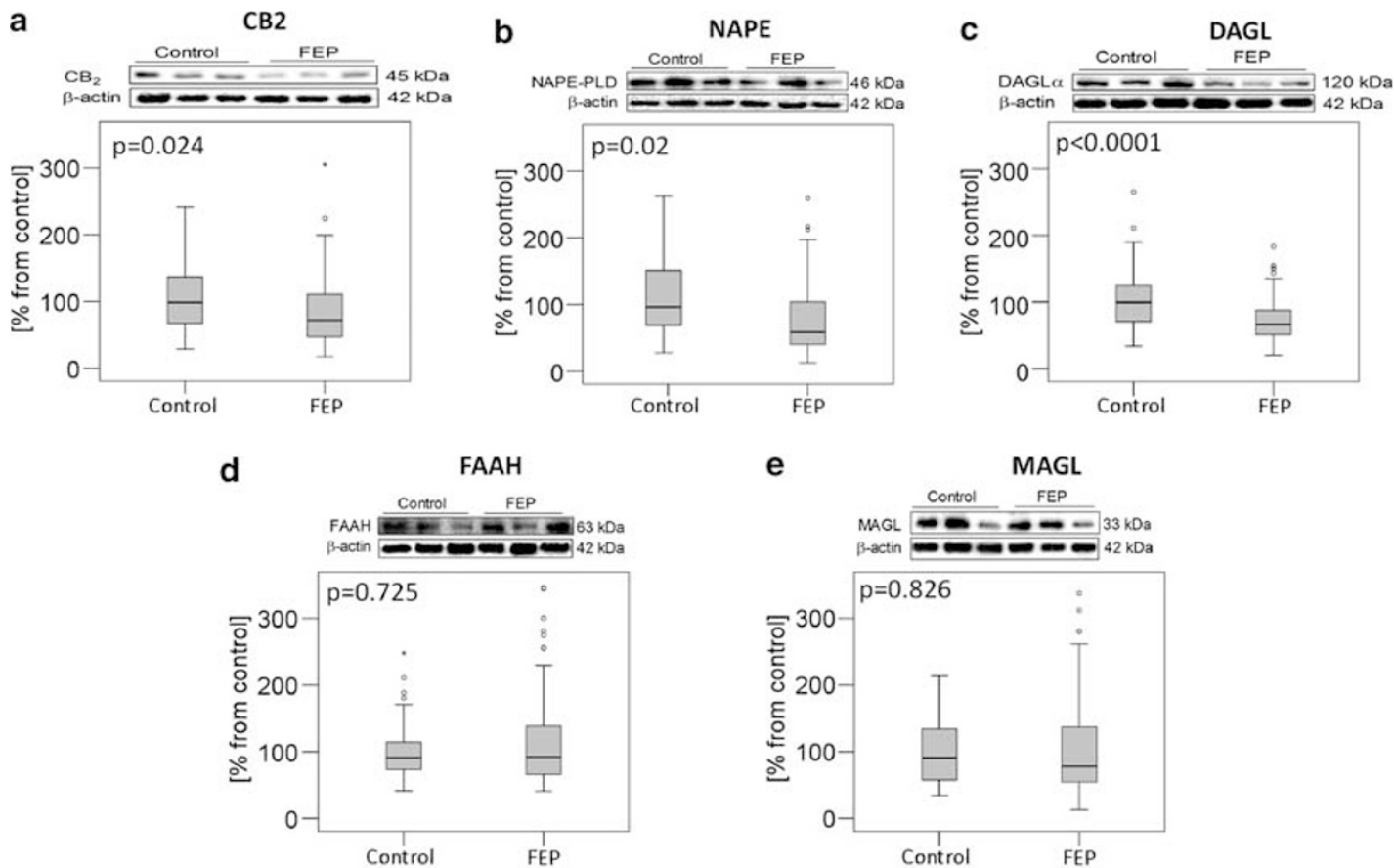

Figure I Western blot analysis of ECS components in PBMC cytosolic extracts from FEP patients and controls. WB analysis (insets) of proteins of interest (upper) and loading control (lower) and densitometric analysis (\% from control). Mean differences (SD) on ECS markers between FEP and controls (univariate analysis). Western blot analysis in PBMC cytosolic extracts from FEP patients and controls of (a) CB2 receptor, (b) NAPE, (c) DAGL $\alpha$, (d) FAAH, and (e) MAGL. Two-tailed t-test was assessed for CB2, NAPE, and DAGL, and for the rest of variables, two-tailed nonparametric Mann-Whitney U-test was used. ${ }^{\circ}$ Represents an atypical value and ${ }^{*}$ an extreme value. 
In the WB analyses carried out in cytoplasmatic extracts, the house keeping gene $\beta$-actin was used as loading control (blots shown in the respective figures). In the Figure 1, two WBs are presented, representing all the samples studied (in each different gel, $n=3$ per group -control or FEP). The insets were the most representative of statistical AU data after densitometric analysis as stated above. All densitometry results are expressed in percentage from control.

\section{Statistical Analysis}

Differences between control and patient features were assessed using two-tailed $\chi^{2}$-tests (on categorical data) and $t$-tests (on variables with approximately normal distributions, as ECS components were). A multiple linear regression analysis was performed to assess the effect of psychotropic medication and clinical variables. $T$-tests were used to differentiate the ECS component expression in the FEP group according to the diagnosis (affective $v s$ nonaffective psychosis), gender, and active cannabis users. Bivariate analyses were used to find differences according to age, clinical scales scores, chlorpromazine's equivalent antipsychotic dosage, BMI, and number of cannabis cigarettes smoked per month.

To calculate the association between FEP and each ECS marker, we used hierarchical logistic regression models with $\mathrm{FEP} /$ control status as the dependent variable, and we controlled for in a step-wise manner for potential confounders. Given that the expression of the ECS can be modified by aging (Bilkei-Gorzo, 2012), can be different between genders (Battista et al, 2012) and it is related to sustained food intake (Di Marzo et al, 2001), analyses were controlled for age, gender, and BMI. Model 1 included the expression of every ECS marker. Model 2 additionally included age and gender. Model 3 additionally included BMI. Model 4 additionally included cannabis use per month. Only those markers with a significant association $(p<0.05)$ with the FEP group in the previous analyses were selected for the following steps. Logistic regression analyses were again calculated with the same system, and all the ECS components chosen were kept and analyzed together in a new Model 1, Model 2 (adding age and gender), Model 3 (adding BMI), and Model 4 (adding cannabis use per month-final model).

Data were managed and analyzed with the IBM SPSS Statistics v.20 (IBM Corp, 2011).

\section{RESULTS}

\section{Demographic, Clinical, and Cannabis Use Features}

Demographic, clinical, and cannabis use information are presented in Table 1 and Supplementary Data 1. Patient and control groups did not differ in gender, age, ethnic group, and socioeconomic status.

The antipsychotic mean dose was of $357.33 \mathrm{mg} /$ day of chlorpromazine equivalents. According to the linear regression analysis, none of the ECS component expression was modified by the use of antipsychotic medication or lithium. We did not find any significant difference between those
Table I Demographic, Clinical, and Cannabis use Features

\begin{tabular}{|c|c|c|}
\hline Characteristic & $\begin{array}{l}\text { Controls } \\
(\mathrm{N}=90)\end{array}$ & $\begin{array}{l}\text { Patients } \\
(\mathrm{N}=95)\end{array}$ \\
\hline \multicolumn{3}{|l|}{ Demographic characteristics } \\
\hline Age (years) & $25.30 \pm 6.41$ & $23.59 \pm 5.60$ \\
\hline \multicolumn{3}{|l|}{ Gender, no. (\%) } \\
\hline Male & $62(68.9)$ & $67(70.5)$ \\
\hline Female & $28(31.1)$ & $28(29.5)$ \\
\hline \multicolumn{3}{|l|}{ Socioeconomic status, no. (\%) } \\
\hline High & $14(15.56)$ & $19(20)$ \\
\hline Medium-High & $14(15.56)$ & $10(10.53)$ \\
\hline Medium & $45(50)$ & $37(38.95)$ \\
\hline Medium-Low & $15(16.67)$ & $23(24.21)$ \\
\hline Low & $2(2.22)$ & $6(6.32)$ \\
\hline \multicolumn{3}{|l|}{ Ethnic group, no. (\%) } \\
\hline Caucasian & $83(92.22)$ & $90(94.74)$ \\
\hline Gipsy & 0 & I (1.05) \\
\hline Maghrebian & 0 & । (1.05) \\
\hline Asian & 0 & । $(1.05)$ \\
\hline Caribbean & 0 & 0 \\
\hline Hispanic & $5(5.56)$ & $2(2.11)$ \\
\hline Others & $2(2.22)$ & 0 \\
\hline Body mass index & $23.30 \pm 3.16$ & $24.93 \pm 5.33^{a}$ \\
\hline Cannabis, active users, no. (\%) & $8(10.53)$ & $16(16.84)$ \\
\hline Cannabis, age of use (years) & $16.54 \pm 2.02$ & $16.04 \pm 2.84$ \\
\hline Cannabis, use per month—cigarettes & $1.33 \pm 6.83$ & $12.67 \pm 35.76^{a}$ \\
\hline Cannabis, time of use (years) & $0.51(2.36)$ & $2.72 \pm 3.84^{\mathrm{a}}$ \\
\hline Cannabis, lifetime contact, no. (\%) & $20(26.32)$ & $52(54.74)^{a}$ \\
\hline $\begin{array}{l}\text { Cannabis, lifetime abuse/dependence, } \\
\text { no. (\%) }\end{array}$ & $5(6.58)$ & $46(48.42)^{a}$ \\
\hline Cannabis, unknown use habits, no. (\%) & $14(15.55)$ & $0(0)$ \\
\hline \multicolumn{3}{|l|}{ Diagnosis, no. (\%) } \\
\hline Affective psychosis & - & $16(16.8)$ \\
\hline Non-affective psychosis & - & $79(83.2)$ \\
\hline \multicolumn{3}{|l|}{ Psychopathology score } \\
\hline \multicolumn{3}{|l|}{ PANSS } \\
\hline Total & - & $53.75 \pm 18.303$ \\
\hline Positive & - & $11.13 \pm 5.693$ \\
\hline Negative & - & $14.85 \pm 6.154$ \\
\hline General & - & $27.77 \pm 8.856$ \\
\hline Young Mania Rating Scale & - & $1.67 \pm 4.457$ \\
\hline $\begin{array}{l}\text { Montgomery-Asberg Depression Rating } \\
\text { Scale }\end{array}$ & & $6.58 \pm 6.718$ \\
\hline Overall functioning score (GAF) & - & $66.39 \pm 13.153$ \\
\hline \multicolumn{3}{|l|}{ Antipsychotic medication, no. (\%) } \\
\hline Risperidone & - & $36(37.9)$ \\
\hline Olanzapine & - & II (| |.5) \\
\hline Aripiprazole & - & $9(9.5)$ \\
\hline Paliperidone & - & $7(7.4)$ \\
\hline Clozapine & - & $7(7.4)$ \\
\hline Quetiapine & - & $5(5.3)$ \\
\hline Ziprasidone & - & $2(2.1)$ \\
\hline None ${ }^{b}$ & - & $18(18.9)$ \\
\hline Lithium use, no. (\%) & - & $9(9.47)$ \\
\hline
\end{tabular}

${ }^{a}$ T-test, $p$ value $<0.05$. Results are based on two-sided tests assuming equal variances with significance level 0.05 . For each significant pair, the key of the smaller category appears under the category with larger mean. Tests are adjusted for all pairwise comparisons within a row of each innermost subtable using the Bonferroni correction.

${ }^{b}$ Includes both never treated patients and those who had stopped the antipsychotic treatment. 
Table 2 Differences in the ECS Markers Expression in FEP and Controls Group (Univariate Model)

\begin{tabular}{|c|c|c|c|c|c|c|c|c|c|}
\hline \multirow[t]{2}{*}{ ECS marker } & \multirow[t]{2}{*}{ Controls $(N=90)$} & \multirow[t]{2}{*}{ Patients $(N=95)$} & \multicolumn{2}{|c|}{ Univariate model } & \multicolumn{5}{|c|}{ Multivariable model } \\
\hline & & & Statistics & $P$-value & B & SE & Wald & OR $(95 \% \mathrm{Cl})$ & $P$-value \\
\hline NAPE & $102.84 \pm 59.64$ & $77 \pm 51.05$ & $t=3.17$ & 0.02 & -0.012 & 0.004 & 7.824 & $0.988(0.980-0.996)$ & 0.005 \\
\hline DAGL & $104.10 \pm 41.86$ & $72.87 \pm 32.36$ & $t=5.69$ & $<\mathbf{0 . 0 0 0 I}$ & -0.032 & 0.007 & 20.041 & $0.969(0.955-0.982)$ & $<0.001$ \\
\hline
\end{tabular}

Association of the ECS components analyzed together and adjusted for age, gender, body mass index, and cannabis use per month (Multiple Logistic Regression Analysis). Bold values indicate significant results $(P<0.05)$.

FEP subjects treated with antipsychotic and those who were not taking antipsychotic treatment $(n=18)$.

Sixteen patients $(16.8 \%)$ were oriented as affective disorders (unipolar major depression or bipolar disorder) with psychotic features.

It was determined that 46 out of the 95 FEP patients met criteria of cannabis abuse or dependence (48.4\%, FEP CAN + ), whereas 49 did not $(51.6 \%$, FEP CAN - ). These subgroups did not statistically differ in the clinical evaluation, the duration of untreated psychosis, the age of onset, and the BMI (Supplementary Data 2).

\section{ECS in Control and FEP Groups}

The $\mathrm{WB}$ analysis revealed a significant lower expression of the $\mathrm{CB} 2$ receptor and of the two main enzymatic sources of endocannabinoids, NAPE and DAGL, in PBMC from FEP group than in the control subjects (Table 2 and Figure $1 \mathrm{a}-\mathrm{c}$, respectively). The expression of the two main degradation enzymatic pathways, FAAH and MAGL, trend to be increased in FEP patients but they did not reach statistical significance in the univariate analysis (Table 2 and Figure $1 \mathrm{~d}$ and e, respectively). After controlling for possible confounders (age, gender, BMI, and number of cannabis cigarettes per month), four of the five components of the ECS studied were significantly associated with FEP. Although univariate analysis showed CB2 differences between groups, these differences were no longer statistically significant after covarying for BMI, cannabis use per month, and the rest of the ECS components.

Among the ECS synthesis enzymes, DAGL had the lowest OR observed $(\mathrm{OR}=0.969)$, meaning that for each decrease in one unit of DAGL expression the probability for suffering a FEP increased in a $3.1 \%\left[\left(\mathrm{e}^{0.084 \times 1}-1\right) \times 100\right]$ (Table 2 and Supplementary Data 3).

When analyzing the ECS degradation enzymes, the highest $O R$ observed was for the FAAH $(O R=1.015)$, meaning that for each unit of this biomarker, the risk of a FEP increased by $1.5 \%\left[\left(e^{0.489 \times 1}-1\right) \times 100\right]$ after controlling for remaining ECS components and all possible studied confounders. Similarly, the results were $1 \%$ for MAGL.

Unfortunately, in our experimental conditions it was not possible to reliably detect $\mathrm{CB} 1$ receptor expression in $\mathrm{PBMC}$ in the great majority of samples.

\section{ECS in Different Clinical Subgroups}

The mean expression of the FAAH of the FEP subjects was significantly higher in men than in women (124.36 vs 87.42, $p=0.014)$. We found a negative correlation between the FAAH expression and total PANSS scores $(p=0.019)$. FAAH expression also showed a negative correlation with the MADRS scale $(p=0.032)$.

According to the diagnosis, patients were classified as non-affective or affective psychosis. There were no differences in the ECS components among these groups. These groups were different in the clinical scale scores and the antipsychotic mean dose, as expected.

Age did not correlate with any of the ECS components studied. The group of patients younger than 18 years old was not different in any of ECS components studied in comparison to the older patients.

\section{Differences between FEP Cannabis Heavy Users/Non-Heavy Users and Controls}

Considering the potential confounding effect of prolonged cannabis use on the peripheral ECS expression, the FEP group was divided in two subgroups according to prolonged use of cannabis, defined as those subjects that had fulfilled DSM-IV criteria for dependence or abuse (at least during 12 consecutive months) throughout their lives. These two groups (FEP CAN + vs FEP CAN - ) were compared with the healthy control group $(n=71)$, excluding from these analyses those volunteers who had presented heavy cannabis use in the past $(n=5)$ and those without complete information of cannabis use $(n=14)$.

One-way ANOVA revealed statistically significant differences between the three diagnostic groups (FEP CAN +, FEP CAN - and control) on CB2 $(F=3.86, p=0.023)$, NAPE $(F=5.47, p=0.005)$, and DAGL $(F=18.35, p<0.001$, Figures 2 and 3). Bonferroni post hoc analysis indicated that FEP CAN + patients had a significant lower expression of CB2 compared with controls (76.72 \pm 41.93 vs $100.65 \pm$ 5.1 AU, $p=0.036$; Figure 2a), lower NAPE expression $(71.64 \pm 48.20$ vs $105.75 \pm 65.05 \mathrm{AU}, p=0.006$; Figure 2b), and lower DAGL expression (69.29 \pm 25.97 vs $108.01 \pm$ 43.21 AU, $p<0.001$; Figure 2c). In this analysis, we found no statistically significant differences between the three groups in the FAAH and MAGL expression. Interestingly, there were no differences between FEP CAN + and CAN groups in the expression of any of the ECS elements studied. 

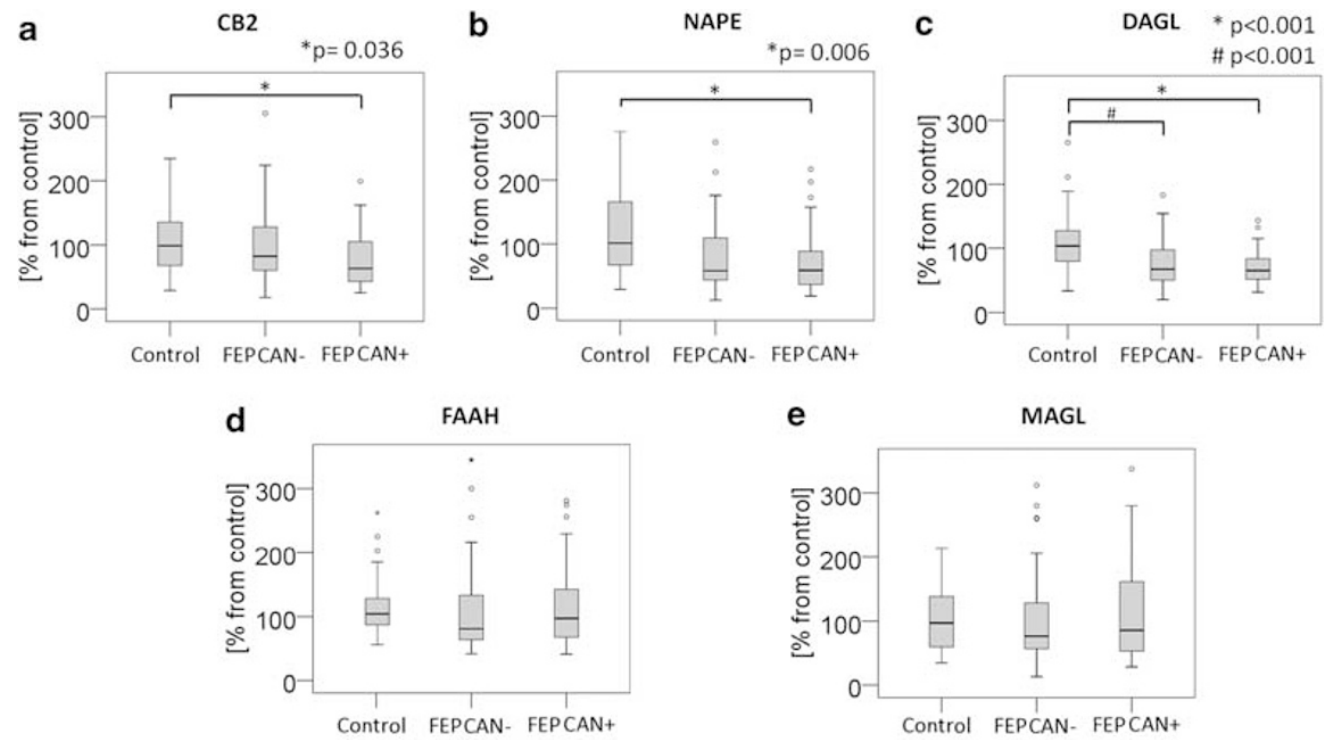

Figure 2 Western blot analysis of the ECS components in PBMC cytosolic extracts from FEP patients (divided according to prolonged cannabis users/ non-users) and controls. Densitometric analysis (\% from control). Mean differences (SD) on ECS markers between FEP cannabis non-users (FEP CAN - ), FEP cannabis users (FEP CAN + ), and controls (univariate analysis). Western blot analysis in PBMC cytosolic extracts from FEP patients and controls of (a) CB2 receptor, (b) NAPE, (c) DAGL $\alpha$, (d) FAAH, and (e) MAGL. Two-tailed $t$-test was assessed for CB2, NAPE, and DAGL, and for the rest of variables, two-tailed nonparametric Mann-Whitney U-test was used. ${ }^{\circ}$ represents an atypical value and ${ }^{*}$ an extreme value.

These results did not change significantly using a semiquantitative index for a better characterization of the cannabis use habit (considering its level and duration) nor by analyzing the ECS results according to the early onset of the cannabis use disorder.

\section{DISCUSSION}

In this study, we found a reduced expression of the CB2 receptor and of the main endocannabinoid synthesis enzymes in PBMC of patients with a FEP compared with matched healthy controls. After controlling for possible confounders, the group of FEP showed a significantly reduced expression of the endocannabinoid synthesis enzymes and an increased expression of the degradative ones. All together, these results describe, for the first time to our knowledge, a dysregulation of these ECS components in patients who have suffered a FEP (Figure 3). Taking into account that prolonged cannabis use is a risk factor to develop a psychotic disorder (Moore et al, 2007; Torrey et al, 2012), the FEP group was subdivided for further statistical analyses. The patient subgroup with a history of heavy cannabis use showed a lower $\mathrm{CB} 2$ receptor expression, NAPE and DAGL expression in comparison to the control group. No statistically significant differences were found with the sporadic/non-users subgroup of patients.

Data reported so far indicate a dysregulation in the ECS (both in terms of ligands and receptors) in patients with schizophrenia and in animal models of psychosis (OrtegaAlvaro et al, 2011; Zamberletti et al, 2012). Our results agree with the described relationship between a diminished CB2 function (polymorphism Q63R) and an increased susceptibility to schizophrenia (Ishiguro et al, 2010), although from our data we cannot ensure whether its reduced expression is previous or concomitant to the psychotic episode. The ECS has been implicated as a neuroprotective system activated in certain neurodegenerative and neuroinflammatory damage (Wolf et al, 2008; Zoppi et al, 2011). The synthesis of endocannabinoids could be a defense mechanism adopted by the brain in a psychotic state (Giuffrida et al, 2004; Giuffrida and Seillier, 2012). A lower expression of CB2 in the group of FEP might indicate a loss of this protector system.

Leweke et al (1999) found elevated AEA levels in the CSF of patients with schizophrenia. Later studies also described elevated CSF AEA levels in antipsychotic-naive first-episode paranoid schizophrenia subjects and in prodromal states of psychosis, with no changes in serum levels (Giuffrida et al, 2004; Koethe et al, 2009). At peripheral level, De Marchi et al (2003) found elevated AEA levels in blood from a small sample of patients with acute schizophrenia. Clinical remission was accompanied by a significant drop in the AEA levels and in the mRNA transcripts for $\mathrm{CB} 2$ and FAAH, suggesting that during the acute phase of schizophrenia the ECS signalling might be altered, not only in the CNS but also at systemic level (De Marchi et al, 2003).

In addition to schizophrenia, several studies in neuroinflammatory diseases (multiple sclerosis, Huntington's, and Parkinson's diseases) have described ECS alterations in the CNS as well as in PBMC (Centonze et al, 2008; Hillard et al, 2012). Non-neuronal cell populations have an active and contributory role in the pathogenesis of these neurodegenerative disorders (Perry et al, 2007), and signs of neuroinflammation can be detected preceding neuronal loss (McGeer et al, 1988). In these CNS pathologies, it can be expected that endocannabinoid concentrations in the circulation and brain are in equilibrium (Hillard et al, 2012), PBMC being a mirror of the CNS endocannabinoid status (Centonze et al, 2008). There is also evidence that AEA and 2AG concentrations are increased at the peripheral circulation when the immune system is activated during 


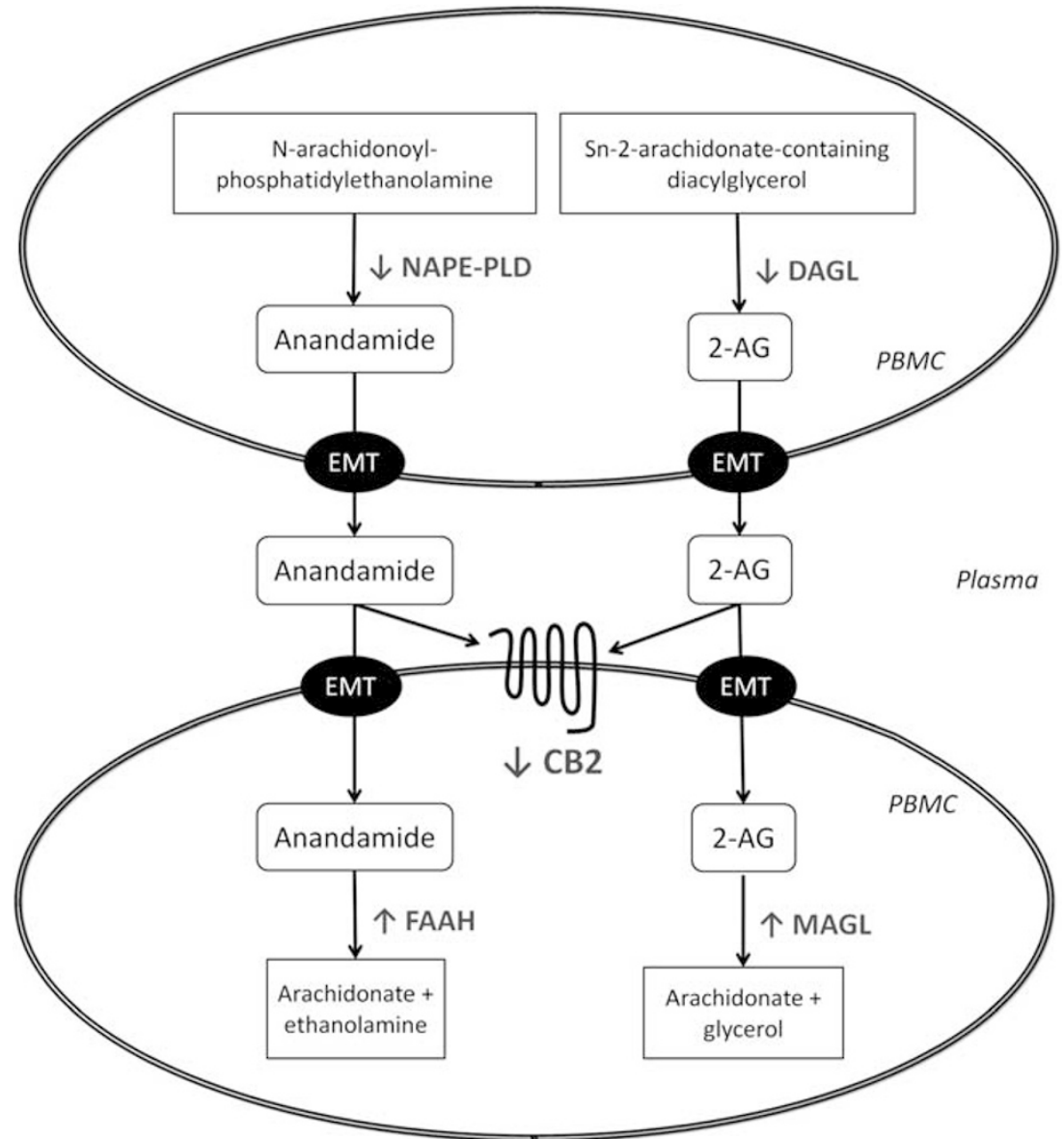

Figure 3 Endocannabinoid system dysregulation in peripheral blood mononuclear cells of patients who have suffered a first episode of psychosis. PBMC, peripheral blood mononuclear cells; NAPE-PLD, N-acyl phosphatidylethanolamine phospholipase; DAGL, diacylglycerol lipase; 2-AG, 2-arachidonoylglycerol; EMT, endocannabinoid membrane transporter; CB2, cannabinoid receptor 2; FAAH, fatty acid amide hydrolase; MAGL, monoacylglycerol lipase. In gray: ECS components studied; $\uparrow:$ higher expression than healthy controls; $\downarrow$ : lower expression than healthy controls.

inflammation, infection, or injury (Hillard et al, 2012). Finally, a recent review suggests a strong association between inflammatory processes and magnetic resonance imaging anomalies in the brain of subjects with schizophrenia, Alzheimer's disease, or major depressive disorder (Frodl and Amico, 2013).

Some of our findings suggest the value of the determination of peripheral ECS components to obtain potential biomarkers for FEP (Hillard et al, 2012). The identification of accessible blood biological markers for psychotic disorders is one of the main needs for both patients and psychiatrists for early diagnosis and evolution monitoring (Schwarz et al, 2012), maybe being the best option to approach to their cerebral expression (Rollins et al, 2010). Taking into account the results of our multivariable analysis, a low expression of DAGL and NAPE or a high expression of FAAH and NAPE would be associated to a highest risk of suffering a FEP. We will be in good position to establish the predictive value of these findings, as this cohort is being followed during a 2-year period (Bernardo et al, 2013).

FAAH expression was higher in men compared with women. It was expected to find differences in FAAH expression between genders, as its activity is regulated by sexual hormones (mainly progesterone and estrogens; Battista et al, 2012; Lazzarin et al, 2004). Knowing that males have a higher lifetime risk of developing schizophrenia (with a male-female relative risk of about 1.4 (Aleman et al, 2003; McGrath et al, 2004)), the different FAAH expression found in this study could be one of the factors involved in these risk differences between genders.

We also found a negative correlation between FAAH expression and PANSS and MADRS score. It seems that more severe, acute patients (with higher PANSS scores) would have higher AEA levels (and lower FAAH expression), suggesting that AEA elevation in acute psychosis may reflect a compensatory adaptation to the disease state (Giuffrida et al, 2004). In our sample, the mean PANSS score was 53.75 (Table 1), pointing to the fact that the majority of the patients were under remission. This could be the reason why FAAH expression is higher in the FEP group. All together, these findings highlight the variations that the ECS could present depending on the state of the psychotic disorder, severity, or presence of depressive symptoms.

The pharmacological manipulation of the ECS may be a novel therapeutic target for the treatment of psychotic disorders. A recent study has shown that cannabidiol moderately inhibits the degradation of AEA, reducing psychotic symptoms of schizophrenia (Leweke et al, 2012). Pharmacological blockade of AEA degradation attenuates induced psychotic-like behaviors in animal 
models (Beltramo et al, 2000; Seillier et al, 2010). The classic focus on CB1 and CB2 has shown the complexity and versatility of the hypothetical ECS role in psychotic disorders (Giuffrida and Seillier, 2012). A recent clinical trial with the CB1 receptor antagonist/inverse agonist rimonabant for improving neurocognition in schizophrenia did not reported clear positive results (Boggs et al, 2012). There are various clinical trials recruiting subjects with schizophrenia to test the utility of cannabidiol (ClinicalTrials.gov identifiers: NCT00588731, NCT00309413, and NCT00916201) and AVE1625 (a potent, selective CB1 antagonist. NCT00439634.)

When controlling for other variables, the association between CB2 and the case-control group stopped being significant, and the association between group and FAAH and MAGL became significant (Table 2). These changes appeared when controlling each ECS component between each other, whereas age, gender, BMI, and active cannabis use did not change much these results (Supplementary Data 3). Thus, these changes were caused by the interaction of the ECS between themselves, which seems logical being part of the same biological system.

Some limitations in this study should be noted. First, CB1 receptor expression was undetectable in almost all samples of PBMC. Although both receptors have a role to restore homeostasis mechanisms, CB1 receptors perform their function mainly in the CNS, whereas CB2 receptors do it mainly at the peripheral level. Some authors have reported CB1 expression in human PBMCs, but its levels are much lower than CB2 and considerable work is still needed to define its relevance and regulatory mechanism/s (Klein et al, 2003). Second, $81.1 \%$ of the patients were receiving antipsychotic treatment. In order to control this possible confounding effect, a multiple linear regression analysis (with each ECS element as dependant variable) was carried out with the chlorpromazine equivalents dosing (Gardner et al, 2010). No effect on the level of any of the ECS components was found. In addition, $9 \%$ of patients were taking lithium and, given its broad pharmacological effects, the possibility of being a confounding factor was also taken into account. This association did not modify the results either. Third, $16.8 \%$ of FEP subjects were diagnosed of affective disorders with psychotic features. This subgroup showed no statistically significant differences in the ECS status compared with non-affective psychosis group. Fourth, cannabis use may have a confounding role when determining the peripheral expression of the different components of ECS. Repeated cannabis use in adolescence produces tolerance to cannabinoid-mediated effects, including brain cannabinoid receptors desensitization and downregulation (Lazenka et al, 2012). The altered expression of $\mathrm{CB} 1$ and $\mathrm{CB} 2$ in cannabis smokers has also been described in PBMC (Nong et al, 2002). In our study, we found no significant differences between FEP CAN $+v s$ FEP CAN - subgroups. Bigger subgroups could have shown larger statistically significant differences. Some peripheral endocannabinoids (AEA and oleoylethanolamide) levels are reduced in substance abusers without schizophrenia in comparison to non-abusing schizophrenia subjects (Desfosses et al, 2012). Along with other drug use disorders, cannabis use should be an important issue to manage in future research.
It is worth mentioning as strength of our study that the diagnostic evaluation was performed with a very comprehensive protocol, with strict inclusion-exclusion criteria. This naturalistic design makes the sample much closer to the 'real life' FEP population. Owing to the heterogeneity of schizophrenia as a clinical entity, the FEP subgroup is of great interest because it avoids the effect of confounding variables, such as prolonged antipsychotic treatment or chronicity (Bernardo et al, 2013). Another key feature of this study is that the age of inclusion is wider than in other previous works, including 23 subjects under 18-year old. Apart from this feature, clinical characteristics of the sample were similar to other studies with FEP in our context (Castro-Fornieles et al, 2008; Kahn et al, 2008). In addition, complex statistical analyses were conducted to limit biases in the results described.

Thus, future clinical investigations should describe the ECS status in medication-free samples and explore the therapeutic potentials of different ECS targets such as the degrading enzymes studied here, TRP channels, PPAR receptors, and cannabinoid membrane transporters (Giuffrida and Seillier, 2012). MAGL activity could be involved in the regulation of cognitive function (Chanda et al, 2010). Studies in high-risk populations will allow determining whether the described alterations in the ECS are present before the psychotic episode starts. This knowledge will have relevant implications to understand the physiopathology of psychosis and also for possible therapeutic implications.

In conclusion, this study has identified that the ECS, which under normal conditions is involved in restoring the homeostatic balance after neural stress, inflammation, or cell damage, appears deregulated in PBMC of patients who had suffered a FEP. Continuous cannabis use could accentuate the malfunction of this endogenous protective system. Some of the peripheral components of the ECS could be used as biomarkers of the disorder. The ECS pharmacological modulation is a promising therapeutic target. Such findings warrant greater attention in future investigations and in the translational significance of these data.

\section{FUNDING AND DISCLOSURE}

Study was supported by the Spanish Ministry of Economy and Competiveness, Instituto de Salud Carlos III (ref. ISCIII 2009-2011: PEPs study PI 080208), CIBERSAM (Intramural Projects 2010: Flamm-PEPs study), European Union FEDER, Dirección General de Universidades, Comunidad de Madrid, CANNAB-CM (S2010/BMD-2308), Government of Catalonia, Secretaria d'Universitats i Recerca del Departament d'Economia i Coneixement (2009SGR1295), Esther Koplowitz Center (Barcelona), I + D Madrid (ref. S2010/ BMD-2308 CANNAB to JCL). The authors declare no conflict of interest.

\section{ACKNOWLEDGEMENTS}

We thank M Fe Barcones (Hospital Clínico Universitario, Zaragoza) and Javier Santabárbara (Department of Preventive Medicine and Public Health, University of Zaragoza) for their methodological support. 


\section{Author Contributions}

$\mathrm{MB}$ wrote the first version of the paper and the figures, performed the statistical analyses, managed and analyzed the clinical data and performed some biochemical determinations; KSM-D and BG-B performed biochemical determinations in plasma and cells; the rest of authors collected the biological samples and the clinical data and analyzed the clinical data; JCL coordinated Flamm-PEPs study; MBer coordinated PEPs study. All of the authors contributed to the final version of the paper.

\section{REFERENCES}

Aleman A, Kahn RS, Selten JP (2003). Sex differences in the risk of schizophrenia: evidence from meta-analysis. Arch Gen Psychiatry 60: 565-571.

American Psychiatric Association (1994). DSM-IV: Diagnostic and Statistical Manual of Mental Disorders. 4th edn. American Psychiatric Association: Washington, DC.

Bangalore SS, Prasad KM, Montrose DM, Goradia DD, Diwadkar VA, Keshavan MS (2008). Cannabis use and brain structural alterations in first episode schizophrenia-a region of interest, voxel based morphometric study. Schizophr Res 99: 1-6.

Battista N, Meccariello R, Cobellis G, Fasano S, Di Tommaso M, Pirazzi V et al (2012). The role of endocannabinoids in gonadal function and fertility along the evolutionary axis. Mol Cell Endocrinol 355: 1-14.

Beltramo M, de Fonseca FR, Navarro M, Calignano A, Gorriti MA, Grammatikopoulos G et al (2000). Reversal of dopamine D(2) receptor responses by an anandamide transport inhibitor. J Neurosci 20: 3401-3407.

Bernardo M, Bioque M (2010). Schizophrenia: from neurobiology to nosology of mental disorders. Actas Esp Psiquiatr 38(Suppl 3): 15-17.

Bernardo M, Bioque M, Parellada M, Saiz Ruiz J, Cuesta MJ, Llerena A et al (2013). Assessing clinical and functional outcomes in a gene-environment interaction study in first episode of psychosis (PEPs). Rev Psiquiatr Salud Ment 6: 4-16.

Bilkei-Gorzo A (2012). The endocannabinoid system in normal and pathological brain ageing. Philos Trans R Soc Lond B Biol Sci 367: 3326-3341.

Boggs DL, Kelly DL, McMahon RP, Gold JM, Gorelick DA, Linthicum J et al (2012). Rimonabant for neurocognition in schizophrenia: a 16-week double blind randomized placebo controlled trial. Schizophr Res 134: 207-210.

Caspi A, Moffitt TE, Cannon M, McClay J, Murray R, Harrington H et al (2005). Moderation of the effect of adolescent-onset cannabis use on adult psychosis by a functional polymorphism in the catechol-O-methyltransferase gene: longitudinal evidence of a gene $\mathrm{X}$ environment interaction. Biol Psychiatry 57: $1117-1127$.

Castro-Fornieles J, Parellada M, Soutullo CA, Baeza I, GonzalezPinto A, Graell M et al (2008). Antipsychotic treatment in child and adolescent first-episode psychosis: a longitudinal naturalistic approach. J Child Adolesc Psychopharmacol 18: 327-336.

Centonze D, Battistini L, Maccarrone M (2008). The endocannabinoid system in peripheral lymphocytes as a mirror of neuroinflammatory diseases. Curr Pharm Des 14: 2370-2442.

Chanda PK, Gao Y, Mark L, Btesh J, Strassle BW, Lu P et al (2010). Monoacylglycerol lipase activity is a critical modulator of the tone and integrity of the endocannabinoid system. Mol Pharmacol 78: 996-1003.

D’Souza DC, Sewell RA, Ranganathan M (2009). Cannabis and psychosis/schizophrenia: human studies. Eur Arch Psychiatry Clin Neurosci 259: 413-431.
De Marchi N, De Petrocellis L, Orlando P, Daniele F, Fezza F, Di Marzo V (2003). Endocannabinoid signalling in the blood of patients with schizophrenia. Lipids Health Dis 2: 5 .

Desfosses J, Stip E, Bentaleb LA, Lipp O, Chiasson JP, Furtos A et al (2012). Plasma endocannabinoid alterations in individuals with substance use disorder are dependent on the "Mirror Effect" of schizophrenia. Front Psychiatry 3: 85.

Di Marzo V, Goparaju SK, Wang L, Liu J, Batkai S, Jarai Z et al (2001). Leptin-regulated endocannabinoids are involved in maintaining food intake. Nature 410: 822-825.

Eggan SM, Hashimoto T, Lewis DA (2008). Reduced cortical cannabinoid 1 receptor messenger RNA and protein expression in schizophrenia. Arch Gen Psychiatry 65: 772-784.

Endicott J, Spitzer RL, Fleiss JL, Cohen J (1976). The global assessment scale. A procedure for measuring overall severity of psychiatric disturbance. Arch Gen Psychiatry 33: 766-771.

First MSpitzer RGibbon MWilliams J (eds) (1999). Entrevista Clínica Estructurada Para los Trastornos del eje-I del DSM-IV. Masson: Barcelona.

Frodl T, Amico F (2013). Is there an association between peripheral immune markers and structural/functional neuroimaging findings? Prog Neuropsychopharmacol Biol Psychiatry (http://dx.doi.org/10.1016/j.pnpbp.2012.12.013).

Gage SH, Zammit S, Hickman M (2013). Stronger evidence is needed before accepting that cannabis plays an important role in the aetiology of schizophrenia in the population. F1000 Med Rep 5: 2.

Garcia-Bueno B, Bioque M, Mac-Dowell KS, Barcones MF, Martinez-Cengotitabengoa M, Pina-Camacho L et al (2013). Pro-/anti-inflammatory dysregulation in patients with first episode of psychosis: toward an integrative inflammatory hypothesis of schizophrenia. Schizophr Bull (e-pub ahead of print 13 March 2013).

Garcia-Rizo C, Fernandez-Egea E, Oliveira C, Justicia A, Bernardo M, Kirkpatrick B (2012). Inflammatory markers in antipsychotic-naive patients with nonaffective psychosis and deficit vs. nondeficit features. Psychiatry Res 198: 212-215.

Gardner DM, Murphy AL, O’Donnell H, Centorrino F, Baldessarini RJ (2010). International consensus study of antipsychotic dosing. Am J Psychiatry 167: 686-693.

Giuffrida A, Leweke FM, Gerth CW, Schreiber D, Koethe D, Faulhaber J et al (2004). Cerebrospinal anandamide levels are elevated in acute schizophrenia and are inversely correlated with psychotic symptoms. Neuropsychopharmacology 29: 2108-2114.

Giuffrida A, Seillier A (2012). New insights on endocannabinoid transmission in psychomotor disorders. Prog Neuropsychopharmacol Biol Psychiatry 38: 51-58.

Henquet C, Murray R, Linszen D, van Os J (2005). The environment and schizophrenia: the role of cannabis use. Schizophr Bull 31: 608-612.

Hillard CJ, Weinlander KM, Stuhr KL (2012). Contributions of endocannabinoid signaling to psychiatric disorders in humans: genetic and biochemical evidence. Neuroscience 204: 207-229.

Ho BC, Wassink TH, Ziebell S, Andreasen NC (2011). Cannabinoid receptor 1 gene polymorphisms and marijuana misuse interactions on white matter and cognitive deficits in schizophrenia. Schizophr Res 128: 66-75.

Hollingshead ABRedlich FC (eds) (1958). Social Class and Mental Illness. Wiley: New York.

IBM Corp (2011). IBM SPSS Statistics for Windows. IBM Corp.: Armonk, NY.

Insel TR (2010). Rethinking schizophrenia. Nature 468: 187-193.

Ishiguro H, Horiuchi Y, Ishikawa M, Koga M, Imai K, Suzuki Y et al (2010). Brain cannabinoid CB2 receptor in schizophrenia. Biol Psychiatry 67: 974-982. 
Kahn RS, Fleischhacker WW, Boter H, Davidson M, Vergouwe Y, Keet IP et al (2008). Effectiveness of antipsychotic drugs in firstepisode schizophrenia and schizophreniform disorder: an open randomised clinical trial. Lancet 371: 1085-1097.

Kay SR, Fiszbein A, Opler LA (1987). The positive and negative syndrome scale (PANSS) for schizophrenia. Schizophr Bull 13: 261-276.

Kirkpatrick B (2009). The Concept of Schizophrenia. Rev Psiquiatr Salud Ment 2: 105-107.

Klein TW, Newton C, Larsen K, Lu L, Perkins I, Nong L et al (2003). The cannabinoid system and immune modulation. J Leukoc Biol 74: 486-496.

Koethe D, Giuffrida A, Schreiber D, Hellmich M, Schultze-Lutter F, Ruhrmann S et al (2009). Anandamide elevation in cerebrospinal fluid in initial prodromal states of psychosis. Br J Psychiatry 194: 371-372.

Kokkevi A, Hartgers C (1995). EuropASI: European adaptation of a multidimensional assessment instrument for drug and alcohol dependence. Eur Addict Res 1: 208-210.

Koskinen J, Lohonen J, Koponen H, Isohanni M, Miettunen J (2009). Rate of cannabis use disorders in clinical samples of patients with schizophrenia: a meta-analysis. Schizophr Bull 36: $1115-1130$.

Lazenka MF, Selley DE, Sim-Selley LJ (2012). Brain regional differences in $\mathrm{CB} 1$ receptor adaptation and regulation of transcription. Life Sci 92: 446-452.

Lazzarin N, Valensise H, Bari M, Ubaldi F, Battista N, Finazzi-Agro A et al (2004). Fluctuations of fatty acid amide hydrolase and anandamide levels during the human ovulatory cycle. Gynecol Endocrinol 18: 212-218.

Leweke FM, Giuffrida A, Koethe D, Schreiber D, Nolden BM, Kranaster L et al (2007). Anandamide levels in cerebrospinal fluid of first-episode schizophrenic patients: impact of cannabis use. Schizophr Res 94: 29-36.

Leweke FM, Giuffrida A, Wurster U, Emrich HM, Piomelli D (1999). Elevated endogenous cannabinoids in schizophrenia. Neuroreport 10: 1665-1669.

Leweke FM, Piomelli D, Pahlisch F, Muhl D, Gerth CW, Hoyer C et al (2012). Cannabidiol enhances anandamide signaling and alleviates psychotic symptoms of schizophrenia. Transl Psychiatry 2: e94.

McGeer PL, Itagaki S, Akiyama H, McGeer EG (1988). Rate of cell death in parkinsonism indicates active neuropathological process. Ann Neurol 24: 574-576.

McGrath J, Saha S, Welham J, El Saadi O, MacCauley C, Chant D (2004). A systematic review of the incidence of schizophrenia: the distribution of rates and the influence of sex, urbanicity, migrant status and methodology. BMC Med 2: 13.

Meyer U, Schwarz MJ, Muller N (2011). Inflammatory processes in schizophrenia: a promising neuroimmunological target for the treatment of negative/cognitive symptoms and beyond. Pharmacol Ther 132: 96-110.
Montgomery SA, Asberg M (1979). A new depression scale designed to be sensitive to change. Br J Psychiatry 134: 382-389.

Moore TH, Zammit S, Lingford-Hughes A, Barnes TR, Jones PB, Burke $\mathrm{M}$ et al (2007). Cannabis use and risk of psychotic or affective mental health outcomes: a systematic review. Lancet 370: $319-328$

Nong L, Newton C, Cheng Q, Friedman H, Roth MD, Klein TW (2002). Altered cannabinoid receptor mRNA expression in peripheral blood mononuclear cells from marijuana smokers. J Neuroimmunol 127: 169-176.

Ortega-Alvaro A, Aracil-Fernandez A, Garcia-Gutierrez MS, Navarrete F, Manzanares J (2011). Deletion of CB2 cannabinoid receptor induces schizophrenia-related behaviors in mice. Neuropsychopharmacology 36: 1489-1504.

Perry VH, Cunningham C, Holmes C (2007). Systemic infections and inflammation affect chronic neurodegeneration. Nat Rev Immunol 7: 161-167.

Rollins B, Martin MV, Morgan L, Vawter MP (2010). Analysis of whole genome biomarker expression in blood and brain. Am $J$ Med Genet B Neuropsychiatr Genet 153B: 919-936.

Schwarz E, Guest PC, Rahmoune H, Harris LW, Wang L, Leweke FM et al (2012). Identification of a biological signature for schizophrenia in serum. Mol Psychiatry 17: 494-502.

Seillier A, Advani T, Cassano T, Hensler JG, Giuffrida A (2010). Inhibition of fatty-acid amide hydrolase and $\mathrm{CB} 1$ receptor antagonism differentially affect behavioural responses in normal and PCP-treated rats. Int J Neuropsychopharmacol 13: 373-386.

Shaffer D, Gould MS, Brasic J, Ambrosini P, Fisher P, Bird H et al (1983). A children's global assessment scale (CGAS). Arch Gen Psychiatry 40: 1228-1231.

Sugranyes G, Flamarique I, Parellada E, Baeza I, Goti J, FernandezEgea E et al (2009). Cannabis use and age of diagnosis of schizophrenia. Eur Psychiatry 24: 282-286.

Torrey EF, Bartko JJ, Yolken RH (2012). Toxoplasma gondii and other risk factors for schizophrenia: an update. Schizophr Bull 38: $642-647$.

Volkow ND (2009). Substance use disorders in schizophreniaclinical implications of comorbidity. Schizophr Bull 35: 469-472.

Wolf SA, Tauber S, Ullrich O (2008). CNS immune surveillance and neuroinflammation: endocannabinoids keep control. Curr Pharm Des 14: 2266-2278.

Young RC, Biggs JT, Ziegler VE, Meyer DA (1978). A rating scale for mania: reliability, validity and sensitivity. $B r J$ Psychiatry 133: 429-435.

Zamberletti E, Rubino T, Parolaro D (2012). The endocannabinoid system and schizophrenia: integration of evidence. Curr Pharm Des 18: 4980-4990.

Zoppi S, Perez Nievas BG, Madrigal JL, Manzanares J, Leza JC, Garcia-Bueno B (2011). Regulatory role of cannabinoid receptor 1 in stress-induced excitotoxicity and neuroinflammation. Neuropsychopharmacology 36: 805-818.

Supplementary Information accompanies the paper on the Neuropsychopharmacology website (http://www.nature.com/npp) 